\title{
HUMOR Y GÉNERO: ANÁLISIS DE CONVERSACIONES ENTRE MUJERES ${ }^{1}$
}

\author{
M. BelÉn Alvarado OrTEgA \\ Universidad de Alicante. Grupo GRIALE
}

Recibido: $15 / 09 / 2014$

Aceptado: 24/11/2014

\section{Resumen}

El trabajo que presentamos pretende analizar enunciados conversacionales que contienen humor producidos por mujeres, que, pueden continuar o no el modo humorístico. Este hecho da lugar a diversas estrategias conversacionales que dependen del sexo y del contexto situacional, marcados por el carácter del corpus recogido y por la identidad de género. Para llevar a cabo nuestro objetivo utilizamos el corpus Corpus de conversaciones coloquiales en proceso de digitalización de Briz y el grupo Val.Es.Co. En el análisis observamos que, cuando aparece el humor, las estrategias que utilizan las mujeres para salvaguardar la imagen son diferentes si el humor es continuado o fallido.

Palabras clave: cortesía, humor continuado, humor fallido, estrategias conversacionales.

\section{Abstract}

The article presents an analysis of conversational humoristic utterances in Spanish produced by women. Humor can appear in conversation as a conversational strategy to identify groups that depend on gender identity and situation. In a specific conversational corpus (Corpus de conversaciones coloquiales, Briz and Val.Es.Co. Group), a particular kind of humor made by women, shown by marks and indicators, will be

1. Este trabajo cuenta con la ayuda del proyecto GRE 12-01 «Lengua y Sexo: Uso del humor en hombres y mujeres» y del proyecto FFI2012-30941: «Innovaciones lingüísticas del humor: géneros textuales, identidad y enseñanza del español».

Feminismo/s 24, diciembre 2014, pp. 17-39

DOI: $10.14198 / \mathrm{fem} .2014 .24 .02$ 
discussed. I present an analysis in order to show different strategies used by women to protect their public face, when they use humor, continued or failed.

Keywords: politeness, continued humor, failed humor, conversational strategies.

Feminismo/s 24, diciembre 2014, pp. 17-39 


\section{Introducción}

El propósito de este trabajo es analizar las estrategias de humor verbal que utilizan las mujeres en la conversación para comprobar que su comportamiento difiere según sea humor continuado o humor fallido. La base teórica que sustenta el trabajo es la Teoría General del Humor Verbal (TGHV) propuesta por Attardo y Raskin ${ }^{2}$, y Attardo ${ }^{3}$, y su aplicación al estudio del español que realizan Ruiz Gurillo y Alvarado ${ }^{4}$. Sobre la identidad de género nos basamos en los trabajos de $\mathrm{Hay}^{5}$, que muestra algunas funciones y fases del humor en la conversación; Santaemilla ${ }^{6}$, que recoge las ideas más importantes sobre género, dominio y conversación; Mills ${ }^{7}$, que relaciona género y descortesía; Kotthoff ${ }^{8}$, que muestra la unión entre género y humor; Bell ${ }^{9}$, evalúa las respuestas de humor fallido; $y$, finalmente, Suderland y Litosseliti ${ }^{10}$, que desarrollan la identidad de género en el análisis del discurso.

2. Attardo, Salvatore y Victor Raskin: «Script theory revis(it)ed: Joke similarity and joke representation model». HUMOR: International Journal of Humor Research, 4, 1999, pp. 293-347.

3. Attardo, Salvatore: «A primer for the linguistics of humor». In Raskin, V. (ed.): The primer of humor research. Berlin: Mouton de Gruyter. 2008.

4. Ruiz Gurillo Leonor y Belén Alvarado Ortega (eds.).: Irony and Humor: From Pragmatics to Discourse. Amsterdam. John Benjamins.2013.

5. Hay, Jennifer: «Functions of humor in the conversation of men and women». In Journal of Pragmatics, 32. 2000, pp. 709-742.

Hay, Jennifer: «The pragmatics of humor support». Humor, 14-1, 2001, pp. 55-82.

6. Santaemilia, José: «Towards a pragmatics of gendered conversation: a few general considerations». En Quaderns de Filología, vol. VII, 2002, pp. 93-113.

7. Mills, Sarah: «Gender and impoliteness». Journal of Politeness Research 1, 2005, pp. 263-280.

8. Kotthoff, Helga: «Gender and humor: The state of the art». Journal of Pragmatics, 38. 2006, pp. 4-25.

9. Bell, Nancy: «Responses to failed humor». Journal of Pragmatics 41, 2009a, pp. 18251836.

Bell, Nancy: «Impolite responses to failed humor». In Humor in interaction, Neal Norrick and Chiaro (eds.), Amsterdan/Philadelphia. John Benjamins, 2009b, pp. 143-163.

10. Suderland Jane y Lia Litosseliti (eds.): Gender identity and discourse analysis. Amsterdan. John Benjamins, 2002. 
El análisis de las estrategias conversacionales de la mujer y el humor se mostrará con ejemplos de humor extraídos del Corpus de conversaciones coloquiales en proceso de digitalización ${ }^{11}$ de Briz y el grupo Val.Es.Co. en los que únicamente ha participado el género femenino. En nuestro corpus hemos analizado 77 minutos de 6 conversaciones coloquiales diferentes, entre mujeres de 18 a 25 años, con algún grado de familiaridad y amistad, y en las que hemos encontrado ocurrencias de humor continuado y de humor fallido.

Por tanto, explicaremos cómo se puede apreciar el humor en la conversación tanto si es continuado como si es fallido (epígrafe 2), lo que nos llevará a establecer diferentes fases. Además, observaremos qué estrategias conversacionales pueden aparecer (epígrafe 3); cómo actúa la mujer en la conversación (epígrafe 4); qué tipo de humor produce (epígrafe 5) y el análisis de ejemplos (epígrafe 6); y, por último, llegaremos a una serie de conclusiones sobre el uso del humor en las conversaciones de mujeres (epígrafe 7).

\section{Humor y conversación: claves para su interpretación}

El humor aparece en la conversación cotidiana de forma espontánea entre familiares y amigos para estrechar lazos entre ellos o bien para atacar la imagen del otro, entre otras razones. Por eso, el humor presenta diferentes funciones en la conversación que han sido consideradas por autores como Attardo, entre otros. De esta manera, cuando utilizamos el humor en la conversación, queremos unirnos a un grupo social, pretendemos mostrar sofisticación, cortesía o, incluso, podemos convencer de algo. Por tanto, el humor presenta en la conversación unas funciones sociales que nos llevan a afirmar que, cuando lo utilizamos, tenemos una intención comunicativa concreta. Esta idea ha sido tratada en Alvarado ${ }^{12}$, trabajo en el que demostramos que el humor sirve, junto con la ironía, para estrechar lazos entre hablante y oyente.

Para saber cómo funciona el humor en la conversación, debemos establecer las dos etapas que menciona Attardo ${ }^{13}$ : por un lado, la capacidad para entender el humor, es decir, la capacidad humorística (humor competence) y, por otro, la actuación del humor (humor performance). La primera fase es la capacidad que tiene el hablante y el oyente de hacer y reconocer el humor en un contexto determinado, mientras que la segunda tiene que ver con el deseo

11. Este corpus se encuentra en proceso de digitalización, y nos gustaría agradecer a A. Briz y al grupo Val.Es.Co. que nos hayan permitido acceder a él.

12. Alvarado, M. Belén: «Una propuesta de estudio para el humor en la conversación coloquial». ELUA, 26, 2012, pp. 7-28.

13. Attardo, Salvatore: Humorous Texts: A semantic and pragmatic analysis. Berlin. Mouton De Gruyter, 2001a, p. 167. 
y la disposición de apreciarlo. De esta manera, hablante y oyente participan activamente en el intercambio comunicativo, a partir de una serie de mecanismos lingüísticos en el enunciado que ayudan al interlocutor a la correcta interpretación del mismo. Esta idea es la que une la TGHV de Attardo con la propuesta del grupo GRIALE que afirmaba que todo intercambio comunicativo humorístico tiene a disposición del oyente indicadores o marcas, es decir, mecanismos lingüísticos bajo la terminología de Attardo, que sirven de pista para interpretar el enunciado de ese modo, en términos irónico-humorísticos. En este trabajo consideramos que tanto hablante como oyente tienen capacidad para apreciar el humor, es decir, la primera fase de humor.

Por tanto, en todos los casos de humor es fundamental observar dónde aparecen los elementos lingüísticos y cuáles son las pistas para que el oyente interprete el enunciado correctamente. Dichas pistas son denominadas por Attardo jab lines o ganchos ${ }^{14}$, si aparecen en la trama, o punch lines o remates, si aparecen al final del texto. Semánticamente son elementos iguales, y la única diferencia que presentan es su posición textual y su función pragmática. De este modo, si aparecen en el centro de la conversación sirven para dar pistas al oyente sobre su intención humorística, es decir, se utilizan como gancho, mientras que si se dan al final se utilizan como remate humorístico. Además, debido al carácter de nuestro corpus, la aparición de las risas ${ }^{15}$ es uno de los fenómenos que vamos a utilizar para el reconocimiento del humor en los enunciados conversacionales, ya que es la consecuencia directa del humor y, por tanto, la manera más precisa de observar que se produce humor en la conversación.

Para comenzar nuestro estudio sobre el humor en las conversaciones de mujeres, partiremos primeramente de la presentación de las fases por las que pasa un enunciado humorístico para producir humor, idea que ha sido tratada por Hay ${ }^{16} y$, posteriormente, por Attardo. Para estos autores dichas fases son: reconocimiento, entendimiento, apreciación y acuerdo, y están estrechamente relacionadas con la capacidad para entender el humor (humor competence) y la actuación del humor (humor performance).

14. Ibíd., p. 82.

15. Sobre este fenómeno numerosos autores han manifestado su opinión al respecto, como Coser (1960), Raskin (1985), Attardo (1993), Hay (2001) o Kotthoff (2003), que han explicado los chistes y las bromas a partir de las risas, y han desarrollado teorías sobre el humor; no obstante, preferimos asumir las ideas de Norrick (1993) sobre las risas, puesto que considera que este signo explícito es el más apropiado para respaldar un corpus de humor como el nuestro.

16. Hay, Jennifer: «The pragmatics of humor support», op. cit., p. 67.

Feminismo/s 24, diciembre 2014, pp. 17-39 
El reconocimiento del enunciado en términos humorísticos es la fase inicial en la que los interlocutores deben interpretar el humor. Esta fase inicial tiene que ver con humor competence, es decir, con la capacidad que tiene el interlocutor de reconocer que en un enunciado hay humor.

La fase de entendimiento está estrechamente relacionada con la anterior, ya que el oyente previamente ha reconocido el enunciado humorístico y, en esta fase, además, lo ha comprendido.

La apreciación es la primera fase que tiene que ver con la actuación del humor, ya que los interlocutores pueden compartir la intención humorística de partida del hablante porque lo han reconocido, lo han entendido y lo han apreciado como tal. En esta fase se decide si se da el humor compartido y continuado o, por el contrario, aparece el humor fallido.

La última fase de un enunciado humorístico es el acuerdo que pueden mostrar tanto oyente como hablante a la hora de producir e interpretar humor. En esta fase de actuación del humor, los interlocutores han decidido continuarlo en la conversación.

No obstante, cabe tener presente que todas estas fases aparecen en la conversación cuando tanto hablante como oyente participan y aceptan el enunciado en términos humorísticos, esto es, se produce un mode adoption por parte de los interlocutores. Según Attardo ${ }^{17}$, mode adoption existe cuando el oyente reacciona al humor con humor, y se dan diferentes intervenciones humorísticas, concepto que también recogen Eisterhold et alii ${ }^{18}$.

Veamos la figura 1 para ilustrar las fases por las que pasa un enunciado humorístico en nuestro corpus. Si observamos la figura, vemos que la competencia para entender el humor envuelve al resto de elementos, puesto que, como hemos dicho anteriormente, para recopilar nuestro corpus partimos de la idea de que todos los interlocutores poseen esa capacidad humorística. De este modo, tras un enunciado humorístico que presente marcas o indicadores (jab lines), es decir, pistas para el interlocutor, el oyente debe ser capaz de interpretarlo de esa manera. Así, estaríamos en la fase de reconocimiento y entendimiento del humor. Tras estas dos primeras fases, encontramos la fase de apreciación que se sitúa a medio camino de la actuación del humor. Esto se debe a que el oyente en este momento es cuando debe decidir si continúa con

17. Attardo, Salvatore: «Humor and irony in interaction: from mode adoption to failure of detection». In L. Anolli, R. Ciceri \& G. Riva (eds.): Say not to say: new perspectives on miscommunication. 2001b, pp. 166-186.

18. Eisterhold, Jodi, Attardo, Salvatore and Boxer, Diana. 2006. «Reactions to irony in discourse: evidence fo the least disruption principle». Journal of Pragmatics 38, 2006, p. 1240 . 


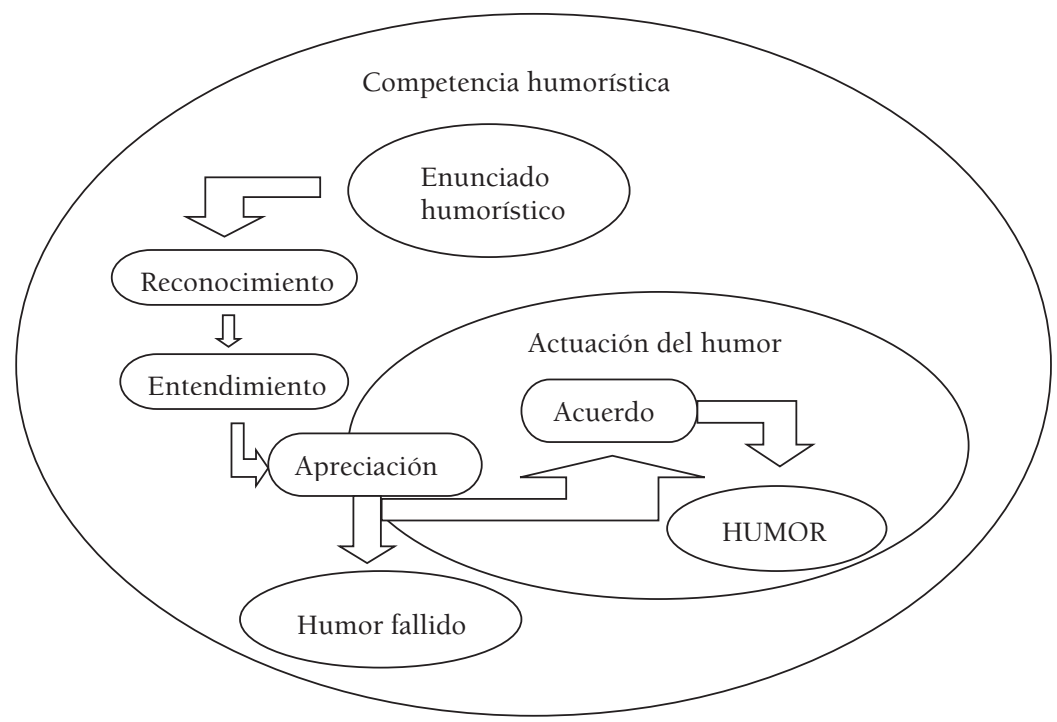

Figura 1. Fases del enunciado humorístico

el modo humorístico (mode adoption) y, por tanto, pasa a la fase de acuerdo y produce o acepta el humor, o, por el contrario, rechaza el humor y no lo continúa. En este caso, estaríamos ante un caso de humor fallido ${ }^{19}$.

Por tanto, la figura 1 sirve, en nuestro trabajo, para ilustrar los casos de humor continuado y humor fallido en la conversación. El humor continuado aparece en las ocurrencias cuando los interlocutores tienen competencia humorística, es decir, reconocen, entienden y aprecian el humor, a partir de los ganchos que les muestra el hablante; y, además, lo comparten, aceptan y continúan con los remates, como pueden mostrar las risas. Mientras que el humor fallido se da cuando los dos interlocutores poseen la capacidad humorística, pero uno de ellos reconoce o entiende un falso gancho y desprecia el humor, sin que haya presencia de remate humorístico. Para entender los conceptos que hemos presentado observemos el ejemplo (1).

En (1) tres amigas conversan sobre el examen práctico del coche que ha aprobado una de ellas, C:

19. Alvarado, M. Belén: «Failed humor in conversational utterances in Spanish: a matter of (im)politeness». En Ruiz Gurillo, L. y Alvarado Ortega, B.: Irony and Humor: From Pragmatics to Discourse. Amsterdam. John Benjamins, 2013. 
(1)

C: pero ¿qué le iba a decir, Mari? //

A: seguro que llega a mi madre ${ }^{20}$ y le dice que diu la xica no sé què d'un cotxe $e^{21}$

C: (risas)

A: quee /s’ha trobat un cot-22 yo qué sé qué historia le montará $\downarrow$ no se entera $\uparrow$ la pobre mujer

C: pero si llamaas/ preguntas por la- / y no le dices algo a ellaa/§

A:

$\S[$ que se ] n'ha anat

en $\operatorname{cootxee} e^{23} \S$

C: $\quad \S[i$ [sabes? Pues]

A:= yo qué sé $\downarrow$ mi madre ¿en coche?

$\mathrm{C}$ : y es cuando le dices [mira $(())]$

B:

[noo pero] se lo pensará/ si le dice algo del

coche $=$

C: [noo]

$\mathrm{B}:=[$ se lo] pensará

(Corpus Val.Es.Co., digital [146.A.:21-32])

En (1) A y C, que son hermanas, crean humor a partir del hecho de que su abuela no reproduce correctamente lo que le cuentan, en este caso, que C ha aprobado el carné de coche. La primera intervención de A da el primer indicador de humor para el oyente, ya que reproduce de forma humorística y con estilo directo las palabras que podría haber dicho la abuela, por tanto, estamos ante el primer gancho; así C reconoce, entiende, aprecia y está de acuerdo con el humor de A, como muestran las risas en su intervención posterior, que actúan como remate. Sin embargo, B no está participando en este momento del humor, puesto que se trata de la abuela de A y C. En su caso, reconoce, entiende y aprecia el humor, pero no lo comparte, como muestra en su intervención, volviendo al tema serio, por lo que B sería un caso de humor fallido.

\section{Estrategias conversacionales y humor}

Parece coherente pensar que la mayoría de las estrategias conversacionales que se utilizan en los casos de humor y de humor fallido están directamente relacionadas con la cortesía y con la imagen de los interlocutores. La cortesía es una estrategia conversacional que se utiliza para evitar conflictos y mantener buenas relaciones entre los individuos de una sociedad, mientras que el humor hace peligrar esa relación cuando se ataca a la imagen de los

20. A y $\mathrm{C}$ son hermanas

21. Val. 'que dice la chica que no sé qué de un coche'

22. Val. 'que se ha encontrado un co (che)'

23. Val. 'que se ha ido en coche'

Feminismo/s 24, diciembre 2014, pp. 17-39 
interlocutores. Brown y Levinson ${ }^{24}$, señalan que el hablante tiene una imagen que debe proteger sin que esto signifique un daño a la imagen de sus interlocutores. En los casos de humor la imagen tanto del hablante como del oyente pueden verse atacadas cuando se convierten en la meta del humor, por lo que es fundamental conocer qué estrategias aplican para defenderse.

Diferentes investigadores enumeran las estrategias que se utilizan en el humor como Culpeper ${ }^{25}$, Hay ${ }^{26}$, entre otros. Tales estrategias están relacionadas con la cortesía y con la descortesía que producen en el intercambio conversacional, como, por ejemplo, la gestión social, la afiliación al grupo conversacional, la sofisticación, la evaluación, la persuasión, la cortesía, la apariencia, la simpatía, la contradicción, el auto-humor, la superposición o simplemente por el mero hecho de contribuir al humor.

Todas estas estrategias probablemente serán diferentes en el uso de hombres y de mujeres y del papel que desempeñan en la conversación. Como el caso que nos ocupa es estudiar el humor en las conversaciones de mujeres, nos centraremos únicamente en las que aparecen documentadas en el corpus analizado $^{27}$. De ahí que, a partir de los datos obtenidos, distingamos para su estudio, como hemos dicho anteriormente, entre el humor que produce la mujer, tanto si es continuado como si es fallido, y concretar qué estrategias se utilizan.

\section{La mujer en la conversación}

La búsqueda de las características del lenguaje femenino ha sido una cuestión que ya interesaba a R. Lakoff, ideas que recoge de sus antecesores ${ }^{28}$. Para estos autores, influenciados por el contexto sociocultural que les rodeaba, la mujer no tiene sentido del humor porque no entiende el remate humorístico de las bromas. Estas ideas, propias del momento en el que se escribe la obra, se deben al papel que tenía la mujer en la sociedad, ya que se situaba en un

24. Brown, Penelope y Stephen Levinson: Politeness: Some universals in language usage. New Cork. Cambridge University Press, 1987, p. 13.

25. Culpeper, Jonathan: «Towards an anatomy of impoliteness». Journal of Pragmatics 25, 1996, p. 355.

26. Hay, Jennifer: «The pragmatics of humor support», op. cit., p. 60.

27. Recordemos que nuestro corpus está extraído de Briz y grupo Val.Es.Co. (corpus digital) del que hemos analizado 77 minutos de 6 conversaciones coloquiales diferentes, en los que las interlocutoras de cada una de las conversaciones son mujeres de entre 18 a 25 años, y en las que hemos encontrado ocurrencias de humor continuado y de humor fallido.

28. Jespersen (1922), Labov (1972), Trudgill (1972), entre otros

Feminismo/s 24, diciembre 2014, pp. 17-39 
segundo plano, dedicada al hogar y a la crianza de sus hijos, y su lenguaje debía ser más decoroso y cuidado que el de los hombres ${ }^{29}$.

Siguiendo esta idea sobre la mujer, Santaemilia ${ }^{30}$ enumera tres factores que influyen en el género y la conversación: el déficit, que tiene que ver con la falta de autoridad que tiene la mujer en la conversación con respecto al hombre; el dominio, que es un privilegio del hombre para poder interrumpir el discurso de la mujer; y la diferencia, que tiene que ver con la visión distinta del mundo que tienen estos dos géneros. Sin embargo, habría que comprobar que estos factores siguen vigentes en la actualidad, ya que parece que estos parámetros no son totalmente válidos, puesto que la mujer está integrada en el mundo laboral, ha adoptado las normas del discurso masculino y las ha hecho propias (interrupciones, lenguaje directo, bromas, etc.). Al tiempo, han evolucionado las teorías sobre el humor en las mujeres, como Suderland y Litosseliti (2002) que desarrollan la identidad de género en el análisis del discurso y en la construcción de papeles sociales caracterizados por la condición social.

En esta misma línea, Kotthoff ${ }^{31}$ muestra la unión entre género y humor, y lo relaciona con el nivel sociocultural. Afirma que las mujeres con nivel sociocultural bajo realizan humor como los hombres, mientras que los hombres con nivel sociocultural alto crean humor como las mujeres. Habría que hacer un estudio contrastivo para comprobar que es así en la conversación, ya que nuestro corpus está basado únicamente en conversaciones en las que solo participan interlocutoras de género femenino con un nivel cultural medio-alto.

Si retomamos la idea de que las mujeres han adoptado el lenguaje de los hombres para hacer humor con un estilo más directo y más agresivo, veremos que se relaciona con la descortesía, ya que su intervención humorística puede afectar a la imagen de los interlocutores. A este respecto, Bell ${ }^{32}$ afirma que algunas bromas pueden actuar sobre la imagen del interlocutor si se utilizan para interrumpir la conversación, puesto que este hecho puede alterar el comportamiento esperado por él. Por eso, para producir humor es necesario empatizar con nuestro interlocutor y saber cómo va a reaccionar ante el enunciado humorístico. Por tanto, cuanto mayor sea el rasgo de familiaridad y cercanía de los interlocutores, mayor será la probabilidad de que aparezca el humor en las conversaciones, ya que los interlocutores que se encuentran en

29. Para un repaso bibliográfico sobre el tema, véase Santaemilia (2002).

30. Santaemilia, J., op. cit., p. 104.

31. Kotthoff, Helga, op. cit., p. 21.

32. Bell, Nancy: «Impolite responses to failed humor», op. cit., p. 158. 
un ambiente distendido se sienten más seguros para producir y recibir humor, tal y como sucede en nuestro corpus.

Observaremos, a continuación, en los ejemplos extraídos de nuestro corpus de mujeres con alto grado de familiaridad y con un nivel de estudios medios y superiores, cómo actúan ante enunciados humorísticos conversacionales. Por eso dedicaremos un epígrafe a mostrar ocurrencias de humor continuado en la conversación, y otro a los ejemplos de humor fallido.

\section{El humor continuado en la conversación de mujeres}

Como hemos dicho, a pesar de que en las investigaciones clásicas del humor predominaba la idea de que las mujeres tenían menos sentido del humor que los hombres ${ }^{33}$, mostraremos, en este epígrafe, que en nuestro corpus las mujeres utilizan el humor continuado de forma frecuente con unas funciones determinadas, ya que se encuentran en un ambiente distendido y familiar. Recordamos que el humor continuado se da cuando se reconoce, se entiende, se aprecia y, además, se comparte y se continúa; es decir, se presenta en los interlocutores la competencia humorística y la actuación del humor, tal y como hemos visto en la figura 1.

Observemos el ejemplo (2), que comparte el mismo contexto que el ejemplo (1). A y C son hermanas y dialogan sobre el hecho de que su abuela no comprenda bien las cosas:

(2)

C: ya pero b- quedamos que- que si llamaba era para- $\mathrm{p}$ (or)- /aprobado

A: mi abuela no se entera tía/ cuando vinieron Jose y todos estos al chalet/ eixos amiguets teus són molt simpàtic ${ }^{34}$, eh? / yo digo ni se le ocurra a nadie decir nada, tía, que s- que lo trabuca todo y no se entera de nada§

C:

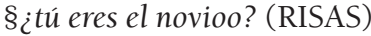

A: (RISAS) además de verdad $\downarrow$ tía

C: y Jose ¿eh?ieh?

A: tu eres el d'Alacant $\downarrow$ el que anaves a casar-te en ella? ? $^{35}$

C: (RISAS) bueno tía $\rightarrow$ se- se lo tengo que decir también al primo Santi// ¿y Jesús cómo ha quedao [yo es que antes no los he visto]

(Corpus Val.Es.Co., digital [146.A.:37-46])

En (2) se producen varias intervenciones humorísticas porque A reproduce a través del estilo directo las palabras que dijo su abuela sobre un amigo (eixos amiguets teus són molt simpàtics) y C continúa con la reproducción (¿itú eres el

33. Hay, Jennifer, op. cit., p. 712.

34. Val. 'esos amiguitos tuyos son muy simpáticos'

35. Val. '¿tú eres el de Alicante, el que ibas a casarte con ella?

Feminismo/s 24, diciembre 2014, pp. 17-39 
novioo?) provocando las risas de ambas interlocutoras. Si observamos el ejemplo, el humor continúa varias intervenciones más, que actúan como gancho humorístico, ya que la meta del humor es la abuela de ambas, que se encuentra ausente en la conversación, por lo que A y C están afianzando sus lazos en el grupo conversacional. Además, como se trata de la abuela de ambas, se encuentran con autoridad y poder suficientes para poder continuar el humor, hecho que contrasta con $\mathrm{B}$, ya que a pesar de que se está presente en la misma conversación, entiende el humor pero no participa del mismo para no atacar la imagen de la abuela de A y C, tal y como hemos visto en (1).

Sin embargo, cuando el humor no se produce hacia la abuela de A y C, B sí que participa del mismo. Veamos (3):

C: $\quad$ Seh- ese- ese que te he dicho yo que ha suspendido que se lo han dicho seguro $\downarrow$ et- ee- era de teleco $\downarrow$ típico PAVITO/ de estos§

A:

$\S$ mari como [el ingenier ${ }^{36}$ ]

C: $\quad[$ de $(())]$ como el INGENIER

A: $\left[\left(\left(\right.\right.\right.$ Beniferrii/ Beniferrii $\left.\left.\left.{ }^{37}\right)\right)\right]$

C: [con una cara de- de pa-/ de PAVO $\rightarrow$ y nada

A: (RISAS) (María Josés8) ¿te acuerdas cuando fuimos a- a-? ¿eras tú la que viniste(s) conmigo? [eeh (RISAS)]

B: [sí sí si] que no se enteraba

(Corpus Val.Es.Co., digital [146.A.:90-99])

En este caso, la meta del humor es una persona ausente en la conversación, que no tiene que ver con ningún familiar de las interlocutoras, el ingenier de Beniferri, por lo que las tres interlocutoras se sienten con confianza y seguridad para poder hacer humor continuado de una persona ausente, incluso atacando su imagen con varios ganchos (con una cara de pavo// ite acuerdas cuando fuimos a...?). Por eso, B continua también con una intervención que actúa de remate humorístico y apoya el humor que ha realizado C (sí, sí, sí, que no se enteraba) para mostrar la identidad de grupo. La estrategia conversacional que se ha utilizado sigue siendo la afiliación al grupo conversacional.

En (4) dos compañeras de trabajo dialogan sobre el hecho de que un alumno de A le había contado que en Noruega había un alto índice de infidelidad:

36. Vulg. por el cat. enginyer 'ingeniero'.

37. Barrio de Valencia. Aluden a una anécdota relacionada con el ingenier.

38. Nombre de B 
(4)

A: quería decir $\downarrow$ bueno $\downarrow$ del centro de Noruega al NORTE $\uparrow$ el norte de Noruega $\uparrow$ que por lo visto $\uparrow$ sí quee es verdad que en general era maayor la infidelidad $\downarrow$

B: (gracias) ${ }^{39}$

A: la infidelidad de los hombres que de las mujeres $\uparrow$ pero quee deel-

?: [((ial revés $(()) ?))]$

A: [el norte] de Noruega $\uparrow$ que era [al revés $\left.\downarrow^{40}\right]$

$\mathrm{B}$ :

[(RISAS)]

A: = y yo $\uparrow /$ digo bueno $\downarrow$ iy CON QUIÉN?§
$\mathrm{B}$ :
A:
$\S(($ y a que $))[(())]$
[¿y con quién] allí?!

porque pensaba el norte de Noruega ijoder! ee- [más arriba=]

$\mathrm{B}$ :

A: = del Círculo Polar Ártico§

$\mathrm{B}$ :

[con más armarios $\uparrow$ ]

$\S$ el norte de Noruega con los esquimales///

(Corpus Val.Es.Co., digital [218.A.1.:83-96])

En (4) A y B continúan el humor porque A atenta contra una situación, el hecho de que los hombres en Noruega sean infieles cuando hay poca densidad de población. Para ello, A aporta diferentes ganchos (y yo digo bueno iy con quién?// ¿y con quién allí?) que B entiende y acepta como humorísticos, tal y como muestran sus risas, que serían el remate humorístico. En este caso, B continúa el humor (con más armarios) y, de este modo, se afianza la identidad de grupo conversacional.

En ocasiones, el humor atenta contra la imagen de una de las interlocutoras, como observamos en (5), en donde A y B son amigas y están hablando sobre anécdotas conjuntas:

A: el sábado ¿te vienes? me voy yo al pueblo/// a pasarlo bomba

B: uic $^{41} /$ de maravillaa ${ }^{42} \S$

A:

§oh pos no te quejarás guapaa§

B:

$\S$ no yo no me quejo§

A:

te vi en to (da) la noche

B: (RISAS) ¿yo a ti sí hermosa?

A: yo estaba hablaando

B: que tú te fuiste primera ¡eh!

39. Alguien le da algo B y lo agradece.

40. Entre risas.

41. Expresión de incredulidad.

42. Entre risas.

Feminismo/s 24, diciembre 2014, pp. 17-39 
A: yo estuve hablaando§

B:

§algo tenía que hacer§

A:

$\S$ yo soy muy buena

B: ssí claro claro// eso está claro

B: no que no m'apeteix ${ }^{43} \xi$

A:

Suna que es irresistiblee (RISAS)

B: $\mathrm{ss}^{44} /$ a mí me pasa lo mismoo $\downarrow \S$

A:

§que va hombre es broma ¿eh?/ to- todo

esto es bromaa ${ }^{45}$

$$
\text { (Corpus Val.Es.Co., digital [235.A:87-104]) }
$$

En (5) A invita a su amiga B al pueblo donde parece que en el pasado B se lo pasó muy bien, ya que conoció a un chico y dejó a su amiga sola toda la noche. La intervención de A (no te vi en toda la noche) atenta contra la imagen de $\mathrm{B}$, que se lo toma de forma humorística, puesto que se trata de un gancho, pero a la vez B se defiende con otro gancho que ataca a A (¿yo a ti sí hermosa?), de donde se infiere que también ligó esa noche. Como vemos, A no esperaba esta intervención, por lo que, a partir de ahí, A y B continúan con una serie de alternancias de turnos irónicos que actúan como ganchos, siguen el modo humorístico y atentan contra la imagen de ambas (yo soy muy buena// sí claro, claro), hasta que B decide parar el modo humorístico volviendo al tema serio (no que no m'apeteix). Sin embargo, A no continúa esa intervención y vuelve a la ironía y al humor (una que es irresistible), por lo que B está obligada a continuarlo para defender la imagen (a mí me pasa lo mismo). Finalmente, A decide que debe parar el juego y afirma que todo lo que está diciendo es broma para salvaguardar la imagen y la relación de ambas. En este caso, la estrategia conversacional evoluciona desde salvaguardar la imagen negativa de cada interlocutora, para que sea respetada por la otra, hasta la última intervención, en la que se intenta estrechar lazos entre ambas.

En (6) las mismas amigas que aparecen en la ocurrencia anterior, siguen dialogando sobre si se van al pueblo o no:

(6)

B:

A: ${ }^{46}$

Spero si van el miércoles ¿tú vas a ir?

B: ¡¿no por qué?!

43. Val. 'No que no me apetece'.

44. Expresión afirmativa e irónica.

45. Entre risas.

46. Chasquido con función de negación.

Feminismo/s 24, diciembre 2014, pp. 17-39 
A: ¿a qué? ((allí no tengo nada)) ((no)) tía y luego ver allí a la gente $e^{47} \rightarrow / / /$ además no tengo quéstudiar

B: ¿no quieres ver a la gentee ${ }^{48}$

A: no (RISAS)/ es que salgo de mi casa y ya lo veo $\downarrow$ (RISAS)

B: ¡vaya! ¿a quién? ${ }^{49}$

A: no sé

B: tú sabrás

A: al vecino de arriba NO TE DIGO /// (2») no además no/ quiero dedicarme a estudiar $\rightarrow / /$ les estoy convenciendo para ir -el sábado (( )) ¿ivale?

(Corpus Val.Es.Co., digital [235.A:114-126])

En (6) B pregunta a su amiga A si va a ir al pueblo con sus padres, pero A no quiere ir para no ver a un exnovio. Tanto A como B saben a quién se están refiriendo y ambas continúan el modo humorístico; sin embargo, B intenta atacar la imagen de A y le hace preguntas que actúan como ganchos humorísticos para incomodarla (¿no quieres ver a la gente?), a las que A responde irónicamente para defender su imagen (al vecino de arriba no te digo). Es A la que realiza la última intervención en modo humorístico como remate para volver al modo serio, ya que ve peligrar su imagen (quiero dedicarme a estudiar...). En este caso, al igual que en (5), la estrategia conversacional que se utiliza con el humor es salvaguardar la imagen negativa de las interlocutoras.

En algunos casos aparecidos en nuestro corpus, el hablante realiza autohumor para salvaguardar la imagen de un ataque inminente. En (7) las mismas amigas que los ejemplos anteriores dialogan sobre un trabajo de carrera que tienen que terminar:

(7)

A: pesadísimo no además/ luego pasarlo y todo// ((tío)) a mí me cuesta más ((que te den-dejeme)) un trocho- un tocho- ay un trocho

B: (RISAS) UN TRocho (RISAS)

A: hablo de categoría ${ }^{50}$ ya $\downarrow$

B: se t'ha pegao de mí

A: síi (RISAS)/ tanto estudio y luego na de na/// (2») me voy a tumbar aquí un poco porque ya// estoy cansá del todo ¿eh?

(Corpus Val.Es.Co., digital [235.A:147-153])

En (7) el humor se produce cuando A no pronuncia bien la palabra tocho para hacer referencia a la cantidad de papeles que deben escribir. B retoma

47. Risa tímida.

48. Entre risas.

49. Entre risas.

50. Ironía.

Feminismo/s 24, diciembre 2014, pp. 17-39 
las palabras que ha reproducido A como un falso gancho para realizar ironía y humor; sin embargo, A siente que se ataca a su imagen negativa, como muestran las risas de $\mathrm{B}$, e intenta defenderse continuando el humor a través de una intervención irónica que actúa como remate (hablo de categoría). Esta intervención produce humor continuado que decide acabar A con la introducción de un tema serio (me voy a tumbar aquí un poco...). En este caso el humor continuado y el auto-humor sirven como estrategia conversacional de protección de imagen.

Así, en la mayoría de los casos encontrados, el humor se continúa para estrechar lazos entre los interlocutores o para proteger la imagen negativa de los ataques del hablante, en menor medida. En las ocurrencias hemos visto que, cuando los temas no tienen que ver directamente con los interlocutores, hay más posibilidades de que se produzca el modo humorístico y continuado, y, por consiguiente, la afiliación al grupo conversacional; mientras que cuando los temas son referentes a alguno de los interlocutores, el humor continuado se produce para proteger la imagen pública. Sin embargo, esta última estrategia conversacional se multiplica en los casos de humor fallido, puesto que lo más habitual en la conversación es que cuando se ataque a la imagen de un interlocutor, no se acepte ni aprecie el modo humorístico, como veremos seguidamente.

\section{El humor fallido en la conversación de mujeres}

En las ocurrencias de humor fallido en la conversación, los interlocutores interpretan de manera errónea una intervención como un falso gancho, es decir, aparece una intervención con posible indicador o marca de humor, cuando en realidad no lo es. Veamos el ejemplo (8) en el que dos amigas conversan sobre el abuelo de A que quiere vivir solo en Valencia:

(8)

A: él $\downarrow$ solo/ a vivir solo $\downarrow / /$ y entonces $\uparrow[h e m o s$ busca(d)o]

B: $\quad$ [chulito $\uparrow]$ como toda la familia ¿no $?^{51}$

(RISAS)

A: y hemos busca(d)o una- (bueno la buscamos $\downarrow$ el el-)

B: el lunes empezó la mujer ¿no?

A: para que $\uparrow$ - por ejemplo mi madre como se va a trabajar por las mañanas $\uparrow \S$

B:

(Corpus Val.Es.Co., digital [173.B:20-27])

51. Entre risas.

Feminismo/s 24, diciembre 2014, pp. 17-39 
En (8) B interpreta la primera intervención de A (él solo, a vivir solo) como un posible gancho, a partir del cual se siente con confianza para crear humor (chulito, como toda la familia, ¿no?). En este caso, la intervención de B no ha sido continuada por A, ya que el enunciado humorístico ataca a la imagen pública de su abuelo y se produce humor fallido en el hablante, que ha intentado crear humor sobre el abuelo de A. A partir de esa intervención de humor fallido se continúa el tema serio ( $y$ hemos buscado una) para proteger la imagen de una persona ausente y familiar del hablante. Por tanto, el humor se reconoce y se entiende, pero no se acepta ni se comparte.

En otras ocasiones, el humor fallido se produce en el oyente cuando es este el que interpreta un elemento lingüístico o varios como ganchos, y no lo son porque el hablante los ignora y continúa con la conversación en modo serio, como vemos en (9). En este ejemplo, varias interlocutoras dialogan sobre una plaga de pulgas que A tenía en su piso:

(9)

B: [ pero/ ¿para] qué limpiaste tanto $\uparrow$ ?

A: tía/ porque estaba empara[noyá (per)día $\uparrow=$ ]

C:

[(RISAS)]

$\mathrm{A}:=[$ quee $\rightarrow]$

C: [ por los piojos] chica§

A: $\quad$ \& yo soy mogo- mogollón de propensa/ a que me pique todo/ o sea / yo / / me depilo las piernas $\uparrow / y$ tengo unos picoreh que me muero/me baño en la playa $\uparrow / y$ me pican las piernas/muy propensa ¿no $\uparrow ? /$ y un día $\rightarrow$ estamos en el $\rightarrow /$ en el $\rightarrow /$ en el bar $\uparrow$ y me dice Carmen/ tienes una cosa

(Corpus Val.Es.Co., digital [151.B.:11-22])

En (9) A, B y C dialogan sobre la manía que tiene una de ellas a los insectos, sobre todo, a las pulgas y a los piojos. La intervención que produce $\mathrm{A}$ (tia/ porque estaba emparanoyá perdía) es interpretada por $C$ como un gancho humorístico por la interpretación literal que hace $C$ del enunciado, cuando en realidad no es una intervención humorística, porque están teniendo una conversación seria sobre la limpieza de una plaga de pulgas que había en su piso. Por tanto, este falso jab line o gancho hace que $\mathrm{C}$ se tome con humor un enunciado, que no es humorístico, y pone su imagen en peligro con respecto al grupo conversacional, que reconoce y entiende el humor pero no lo comparte ni aprecia. De ahí que $C$ no continúe el humor y opte por el tema serio como modo de reparar su imagen hacia los demás.

El silencio también aparece en nuestro corpus como forma de respuesta ante enunciados potencialmente humorísticos. Este fenómeno ha sido 
estudiado por autores como $\mathrm{Hay}^{52}$, Norrick ${ }^{53}$ o Priego Valverde ${ }^{54}$ que afirman que el silencio se interpreta como falta de acuerdo con el enunciado. Sin embargo, todos advierten de la posibilidad de que el silencio pueda deberse también a la falta de entendimiento del enunciado humorístico, es decir, aparece en los casos en los que no se ha reconocido el humor. Recordemos que tomamos como humor fallido aquellos enunciados que se dan en la conversación coloquial y que han superado las tres primeras fases de humor, reconocimiento, entendimiento y apreciación; por tanto, entendemos que los interlocutores son capaces de distinguir el humor, pero no lo han continuado, tal y como vimos en la figura 1. Así, no tenemos en cuenta los silencios que se dan en las intervenciones humorísticas en las que el oyente no ha entendido el enunciado, hecho que sí sucede en los trabajos de Priego Valverde, entre otros. Observemos el siguiente ejemplo que se produce en la misma conversación que la ocurrencia anterior, en la que varias amigas dialogan sobre la plaga de pulgas que A tuvo en su piso:

A: = superemparanoyada con eso/ pero mogollón/ hasta lo de abajo $\uparrow / /$ paraa/ lo- d'ese todo// después voy/me ca-me pongo el otro pijama que tenía limpio/ me lo pongo/ me acuesto/después/me tocó $\uparrow /$ me pon- y aquí/ yo $\uparrow$ hasta $\rightarrow$ / fíjate tú [lo que hace la psicología=]

C: [(RISAS)]

$\mathrm{A}:$ : que yo/ hasta me notaba erupciones/o sea/ yo me notaba ya/ el bultito de que me habían picaoł/y por la mañana no tenía nada/ y yo me tocaba aquí y tenía/ me tocaba aquí que me rascaba/ y tenía también/ me tocaba aquí/ y también tenía/ mira/ hasta las seis de la ma-tía iqué no dormí! $\uparrow /$ (es) que ¡NO DORMí! ¿eh? $\uparrow /$ no podía dormir $\uparrow /$ en la cama $\rightarrow$ / a punto de llorar/ digo la despierto\$ y hacemos aquí la limpieza a las seis de la mañana// y ya desde ese día/ pues emparanoyá/ mañana limpio la habitación

(Corpus Val.Es.Co., digital [151.B.:11-22])

En (10) A cuenta que sigue obsesionada con la plaga de pulgas que tuvo en su piso y que no le deja hacer su vida normal. C interpreta la primera intervención de A como un falso gancho del hablante (superemparanoyada...); sin embargo, nadie continúa el modo humorístico, porque lo que A está contando es un tema serio, por lo que $\mathrm{C}$ opta por el silencio en el resto de intervenciones

52. Hay, Jennifer, op. cit.

53. Norrick, Neal: «Issues in conversational joking». Journal of Pragmatics 35, 2003, pp. 1333-1359.

54. Priego Valverde, Beatrice: «Failed humor in conversation: A double voicing analysis». In Norrick, Neal and Delia Chiaro. 2009. Humor in interaction, Amsterdam/Philadelphia. John Benjamins, pp. 165-186. 
de A, al igual que el resto de interlocutoras. De este modo, no peligra la imagen pública de ninguna de ellas y se afianza el grupo conversacional. Por ello, el silencio se utiliza como una estrategia conversacional relacionada directamente con la cortesía, puesto que sirve para salvaguardar la imagen del interlocutor, que se ha visto dañada por la emisión o la interpretación de un falso enunciado humorístico como un gancho erróneo, como observamos en (10).

Pero, además del silencio y de la continuación del tema serio, existen otras estrategias conversacionales que se utilizan para salvaguardar la imagen del interlocutor como, por ejemplo, el cambio de tópico conversacional. Veamos (11) en el que las mismas interlocutoras de las ocurrencias anteriores siguen dialogando sobre los planes del próximo verano:

C:

[yo me voy ] a pegar un verano que te cagas $\uparrow$ el invierno va a ser una mierda lo que este verano me voy pegar un viaje por toda Europa que se va a cagar la perra (RISAS) ((ellaa))/ quiero hacer el Interrail ${ }^{55}$ [ con]

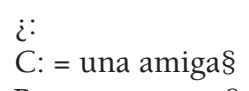

(Corpus Val.Es.Co., digital [151.B.:424-430])

En (11) C habla sobre los planes de verano que se ha propuesto al acabar el curso. Mientras cuenta sus intenciones, utiliza la expresión coloquial se va a cagar la perra como gancho humorístico, que le produce risas a ella misma, pero no a sus interlocutoras, que están más preocupadas de aprobar todas las asignaturas que en pensar en el verano. En este caso, también se produce humor fallido en el hablante, ya que ha introducido un gancho que sus oyentes no han interpretado como tal y no han compartido, porque no consideran la situación humorística. Así, deciden cambiar el tópico conversacional, tal y como muestra la intervención de B (pero ipiensas sacarlo todo en junio?) para que no peligre la imagen negativa de $C$.

En los casos de humor fallido, el oyente interpreta un falso gancho o el hablante introduce ganchos en su intervención que producen incongruencias. Estas pistas, que deja al oyente para que las interprete y continúe el modo humorístico con algún remate o resuelva esa incongruencia con otro gancho, fracasan porque no se aceptan como enunciados humorísticos. Los temas en los ejemplos que hemos analizado de humor fallido tienen que ver con las

55. Se trata de un billete de tren único que te permite viajar por Europa durante un tiempo determinado. Es muy popular entre los jóvenes.

Feminismo/s 24, diciembre 2014, pp. 17-39 
vivencias de las interlocutoras, de ahí que decidan no continuar el humor para proteger su imagen pública.

\section{Conclusiones}

Este trabajo se ha centrado específicamente en analizar los enunciados conversacionales producidos por mujeres en los que aparece el modo humorístico continuado y fallido. Si se percibe el modo humorístico y se comparte se darían las cuatro fases del humor, vistas en la figura 1 , y, por tanto, el humor continuado en el enunciado, mientras que si no se continúa el modo humorístico, porque no hay acuerdo, se dan solo tres fases (reconocimiento, entendimiento y apreciación) y aparecen los casos de humor fallido.

En los casos en los que el grado de familiaridad o cercanía es menor entre las interlocutoras, se producen más casos de humor continuado en mujeres. Este hecho se debe a que se afianzan los lazos con el grupo conversacional y permite proteger la imagen pública de las interlocutoras. Sucede, sobre todo, en los casos en los que se realiza humor con temas relacionados con situaciones o con personas ausentes, como hemos visto en (2), (3), (4), (5), (6) y (7).

El humor fallido en conversaciones de mujeres se produce, en la mayoría de los casos, para proteger la imagen de las interlocutoras, ya que el grado de familiaridad y cercanía favorece la aparición de la ironía y el humor que atenta sobre los participantes del acto comunicativo. Se produce, sobre todo, con temas relacionados con su vida cotidiana. Las estrategias conversacionales que se han utilizado en los casos analizados son: la continuación del tema serio, como hemos visto en (8) y (9), los silencios (10) y el cambio de tópico conversacional (11).

En general, podemos afirmar que las mujeres en la conversación cotidiana realizan humor continuado para afianzar los lazos del grupo conversacional y lo rechazan para salvaguardar su imagen pública. En futuros trabajos observaremos qué sucede cuando son hombres los que realizan el humor.

\section{Referencias}

Corpus

BRIZ, Antonio y Grupo Val.Es.Co.: Corpus de conversaciones coloquiales (digital), 2002.

Bibliografía

Alvarado, M. Belén: «Las marcas de la ironía». In Interlingüística, 16, Online, 2006. 
Alvarado, M. Belén: «Ironía y cortesía». En Ruiz Gurillo L. \& X. Padilla (eds.). Dime cómo ironizas y te diré quién eres: una aproximación pragmática a la ironía. Frankfurt, Peter Lang. 2009, pp. 333-345.

Alvarado, M. Belén: Las fórmulas rutinarias del español: teoría y aplicaciones. Frankfurt, Peter Lang, 2010.

AlvarAdo, M. Belén: «Una propuesta de estudio para el humor en la conversación coloquial». ELUA, 26, 2012, pp. 7-28.

AlvarAdo, M. Belén: «Failed humor in conversational utterances in Spanish: a matter of (im)politeness». En Ruiz Gurillo, L. y Alvarado Ortega, B.: Irony and Humor: From Pragmatics to Discourse. Amsterdam. John Benjamins, 2013.

AtTARDO, Salvatore: Humorous Texts: A semantic and pragmatic analysis. Berlin. Mouton De Gruyter, 2001a.

ATTARDO, Salvatore: «Humor and irony in interaction: from mode adoption to failure of detection». En L. Anolli, R. Ciceri \& G. Riva (eds.): Say not to say: new perspectives on miscommunication, 2001b, pp. 166-186.

ATtARDO, Salvatore: «Multimodal markers of irony and sarcasm», en Humor: International Journal of Humor Research, 16:2, 2003, pp. 243-260.

ATtARDO, Salvatore: «A primer for the linguistics of humor». En Raskin, V. (ed.): The primer of humor research. Berlin, Mouton de Gruyter, 2008.

ATTARDO, Salvatore y Victor RASKIN: «Script theory revis(it)ed: Joke similarity and joke representation model». En HUMOR: International Journal of Humor Research, 4, 1991, pp. 293-347.

BELL, Nancy: «Responses to failed humor», Journal of Pragmatics 41, 2009a, pp. 1825-1836.

BELL, Nancy: «Impolite responses to failed humor». En Humor in interaction, Neal Norrick and Chiaro (eds.), Amsterdan/Philadelphia. John Benjamins, 2009b, pp. 143-163.

BROWn, Penelope y Stephen LeVInSON: Politeness: Some universals in language usage. New Cork. Cambridge University Press, [1978] 1987.

COSER, Rose: «Laughter among colleagues: A study of the social functions of humor among the staff of a mental hospital», Psychiatry 23, 1960, pp. 83-91.

CUlPEPER, Jonathan: «Towards an anatomy of impoliteness», Journal of Pragmatics 25, 1996, pp. 349-367.

EISTERHOlD, Jodi, ATTARDO, Salvatore and BOXER, Diana: «Reactions to irony in discourse: evidence fo the least disruption principle», Journal of Pragmatics 38, 2006, pp. 1239-1256.

HAVERKATE, Henk: «La ironía verbal: análisis pragmalingüístico». En Revista Española de Lingüística, 15, 2, 1985, pp. 343-391.

HAVERKATE, Henk: La cortesía verbal. Estudio pragmalingüístico. Madrid, Gredos, 1994.

Feminismo/s 24, diciembre 2014, pp. 17-39 
HAY, Jennifer: «Functions of humor in the conversation of men and women». En Journal of Pragmatics, 32, 2000, pp. 709-742.

HAY, Jennifer: «The pragmatics of humor support», Humor 14-1, 2001, pp. 55-82.

HolmeS, Janet: «Sharing a laugh: Pragmatic aspects of humor and gender in the work-place». En Journal of Pragmatics, 38, 2006, pp. 26-50.

Holmes, Janet y Meredith MARRA: «Having a laugh at work: how humour contributes to workplace culture». En Journal of Pragmatics, 34, 2002, pp. 1683-1710.

JESPERSEN, Otto: Language: Its nature, development and origins. London. Allen and Unwin, 1922.

KotThoff, Helga: «Gender and joking: on the complexities of women's image politics in humorous narratives». En Journal of Pragmatics, 32, 2000 pp. 55-80.

Kotтhoff, Helga: «Responding to irony in different contexts: on cognition in conversation». Journal of Pragmatics, 35, 2003, pp. 1387-1411.

Kotthoff, Helga: «Gender and humor: The state of the art». En Journal of Pragmatics, 38, 2006, pp. 4-25.

LABOV, William: «The social stratification of $\mathrm{r}$ in New York City Department Stores». En Coupland y Jaworski (eds.): Sociolinguistics: A reader and Coursebook. New York. Martin`s Press, 1997, pp. 168-178.

LAKOFF, Robin: Language and Woman's place. New York, Harper and Row, 1975.

MiLls, Sarah: «Gender and impoliteness». Journal of Politeness Research 1, 2005, pp. 263-280.

NORRICK, Neal: «Issues in conversational joking». Journal of Pragmatics 35, 2003, pp. 1333-1359.

NORRICK, Neal and Delia Chiaro: Humor in interaction. Amsterdam/Philadelphia, John Benjamins, 2009.

PRIEGO VAlVERDE, Beatrice: «Failed humor in conversation: A double voicing analysis». En Norrick, Neal and Delia Chiaro: Humor in interaction. Amsterdam/Philadelphia. John Benjamins, 2009, pp. 165-186.

RASKIN, Victor: Semantic Mechanisms of Humor. Dordrecht, D. Reidel, 1985.

RUIz GuRILlo, Leonor: «¿Cómo se gestiona la ironía en la conversación?» En Rilce, 25.2, 2009, pp. 363-377.

RUIZ GURILLO, Leonor: «Para una aproximación neogriceana a la ironía en español». En RFE, 40, 2, 2010, pp. 95-124.

Ruiz GuRILlo, Leonor: La lingüística del humor en español. Madrid, Arco Libros, 2012.

RuIz GuRILlo, Leonor y Xose Padilla (eds.): Dime cómo ironizas y te diré quién eres: una aproximación pragmática a la ironía. Frankfurt, Peter Lang, 2009.

Ruiz Gurillo, Leonor y Belén Alvarado Ortega (eds.): Irony and Humor: From Pragmatics to Discourse. Amsterdam. John Benjamins, 2013. 
SANTAEMILIA, José: «Towards a pragmatics of gendered conversation: a few general considerations». En Quaderns de Filología, vol. VII, 2002, pp. 93-113.

SudERlAnd, Jane y Lia Litosseliti (eds.): Gender identity and discourse analysis. Amsterdan. John Benjamins, 2002.

SPENCER-OATEY, Helen: (Im)Politeness, face an perceptions of rapport: un packaging their bases an interrelationships». Journal of Politeness Research 1, 2005 pp. 95-119.

TRUdGILL, Peter: The social differentiation of English in Norwich. Cambridge. Cambridge University Press, 1972.

ZAJDMAN, Anat: «Humorous face-threatening acts: Humor as strategy». Journal of Pragmatics 23, 1995, pp. 325-339. 